\title{
Some Inequalities of the \\ Incomplete Gamma and Related Functions
}

\author{
Feng Qi and Jia-Qiang Mei
}

\section{Dedicated to our advisor Prof. Sen-Lin Xu at USTC}

Abstract. In the article, many inequalities of the integrals

$$
\int_{x}^{\infty} \mathrm{e}^{-t^{p}} d t, \quad \int_{0}^{x} \mathrm{e}^{-t^{p}} d t, \quad \int_{0}^{x} \mathrm{e}^{t^{p}} d t
$$

for $p>0$, which are related to the incomplete gamma function, are established. The approach used in the paper could yield more particular inequalities of the above functions. Some known results are generalized, extended or refined.

Keywords: Inequalities, incomplete gamma function, complementary error function AMS subject classiflcation: Primary 33 B 20, secondary 26 D 07, 26 D 15

\section{Introduction}

It is well-known that the incomplete gamma function is defined for $\operatorname{Re} z>0$ by

$$
\Gamma(z, x)=\int_{x}^{\infty} t^{z-1} e^{-t} d t, \quad \gamma(z, x)=\int_{0}^{x} t^{z-1} e^{-t} d t
$$

and $\Gamma(z, 0)=\Gamma(z)$ is called the gamma function, $\Gamma(0, x)=E_{1}(x)$ the exponential integral. Notice that the integral $\int_{x}^{\infty} e^{-t^{p}} d t$ can be expressed in terms of the incomplete gamma function as

$$
\int_{x}^{\infty} e^{-t^{p}} d t=\frac{1}{p} \Gamma\left(\frac{1}{p}, x^{p}\right) \quad(p>0) .
$$

Thus it is important and interesting to study the function $\int_{x}^{\infty} e^{-t^{p}} d t$ and the related functions $\int_{0}^{x} e^{-t^{p}} d t$ and $\int_{0}^{x} e^{t^{p}} d t$ for $p>0$.

Feng Qi: Jiaozuo Inst. Techn., Dept. Math., Jizozuo City, Henan 454000, China; e-mail: qifeng@jzit.edu.cn. The author was partially supported by NSF of Henan Province, China Jia-Qiang Mei: Univ. Sci. \& Techn. of China, Dept. Math., Hefei City, Anhui 230026, China 
In [2], A. Laforgia and S. Sismondi proved some monotonicity properties for the complementary error function

$$
\operatorname{erfc}(x)=\frac{2}{\sqrt{\pi}} \int_{x}^{\infty} \mathrm{e}^{-t^{2}} d t \quad(x>0)
$$

and as a consequence of these they established many inequalities for this function. Using the monotonicity and inequalities of the generalized weighted mean values with two parameters and the extended mean values defined and studied in $[4,8,10]$, the first author [5] found some monotonicity results and inequalities for the gamma and incomplete gamma functions. Among other things, it is proved that the functions

$$
\left(\frac{\Gamma(s)}{\Gamma(r)}\right)^{\frac{1}{s-r}}, \quad\left(\frac{\Gamma(s, x)}{\Gamma(r, x)}\right)^{\frac{1}{a-r}}, \quad\left(\frac{\gamma(s, x)}{\gamma(r, x)}\right)^{\frac{1}{0-r}}
$$

are increasing respect to $r>0, s>0$ and $x>0$. Notice that the monotonicity of the function $\left[\frac{\Gamma(s)}{\Gamma(r)}\right]^{1 /(s-r)}$ respect to $r>0$ and $s>0$ is an old and well-known consequence of the logarithmical convexity of the gamma function $\Gamma$. By the Tchebycheff integral inequality, the first author, L.-H. Cui and S.-L. Xu in [7], inter alia, constructed many inequalities of the error function

$$
\operatorname{erf}(x)=\frac{2}{\sqrt{\pi}} \int_{0}^{x} e^{-t^{2}} d t
$$

and got the lower bound of the function $\int_{0}^{x} e^{-t^{\alpha}} d t$ and the upper bound of the function $\int_{0}^{x} e^{t^{\alpha}} d t$ for $x>0$ and $\alpha>1$. From the Hermite-Hadamard inequality, considering the convexity of the function $e^{t^{p}}$ and $e^{-t^{p}}$, the first author and S.-L. Guo in [9] obtained some inequalities of the functions $\int_{0}^{x} e^{-t^{p}} d t$ and $\int_{0}^{x} e^{t^{p}} d t$ for $x>0$ and $p>0$. More other inequalities for the gamma and incomplete gamma functions could be found in [1, 3].

In this article, motivated by [2] and [9], we further researched the integrals

$$
\int_{x}^{\infty} \mathrm{e}^{-t^{\alpha}} d t \quad(\alpha>0), \quad \int_{0}^{x} \mathrm{e}^{t^{\beta}} d t \quad(\beta>0), \quad \int_{0}^{x} \mathrm{e}^{-t^{\theta}} d t \quad(\theta>0)
$$

for $x>0$, present some monotonicity properties that generalize one of the main results in [2] by A. Laforgia and S. Sismondi, and form some particular inequalities of the above integrals. 


\section{Main theorems}

The following three theorems are elementary and important and they could yield more special results.

Theorem 1. For any given $\alpha>0$, let $h_{\alpha}(x)$ be defined for $x>0$ by

$$
h_{\alpha}(x)=e^{x^{\alpha}} \int_{x}^{\infty} e^{-t^{\alpha}} d t+p_{\alpha}(x)
$$

where $\lim _{x \rightarrow \infty} \mathrm{e}^{-x^{\alpha}} p_{\alpha}(x)=0$ and $p_{\alpha}$ satisfies

$$
p_{\alpha}^{\prime}(x)-\alpha x^{\alpha-1} p_{\alpha}(x)-1>0 \text {. }
$$

Then $h_{\alpha}(x)<0$. If $(4)$ is reversed, then $h_{\alpha}(x)>0$.

Proof. By direct calculation, we have

$$
h_{\alpha}^{\prime}(x)=\alpha x^{\alpha-1} h_{\alpha}(x)-\alpha x^{\alpha-1} p_{\alpha}(x)+p_{\alpha}^{\prime}(x)-1
$$

and

$$
\begin{aligned}
\mathrm{e}^{x^{\alpha}}\left[\mathrm{e}^{-x^{\alpha}} h_{\alpha}(x)\right]^{\prime} & =h_{\alpha}^{\prime}(x)-\alpha x^{\alpha-1} h_{\alpha}(x) \\
& =p_{\alpha}^{\prime}(x)-\alpha x^{\alpha-1} p_{\alpha}(x)-1 \\
& >0 .
\end{aligned}
$$

From this it follows that $\mathrm{e}^{-x^{\alpha}} h_{\alpha}(x)$ increases. Since $\lim _{x \rightarrow \infty} \mathrm{e}^{-x^{\alpha}} p_{\alpha}(x)=0$, we have $\lim _{x \rightarrow \infty} \mathrm{e}^{-x^{\alpha}} h_{\alpha}(x)=0$, hence $h_{\alpha}(x)<0$. The proof of Theorem 1 is completed

Theorerm 2. Let $g_{\beta}(x)$ be defined for $x>0$ by

$$
g_{\beta}(x)=\mathrm{e}^{-x^{\beta}} \int_{0}^{x} \mathrm{e}^{t^{\rho}} d t+q_{\beta}(x)
$$

where $\lim _{x \rightarrow 0} q_{\beta}(x)=0$ and $\beta>0$ is any given number. If $q_{\beta}$ satisfies

$$
q_{\beta}^{\prime}(x)+\beta x^{\beta-1} q_{\beta}(x)+1>0
$$

then $g_{\beta}(x)>0$. If $(6)$ is reversed, then $g_{\beta}(x)<0$.

Proof. Easy calculation yields

$$
g_{\beta}^{\prime}(x)=-\beta x^{\beta-1} g_{\beta}(x)+\beta x^{\beta-1} q_{\beta}(x)+q_{\beta}^{\prime}(x)+1
$$

and

$$
\begin{aligned}
{\left[\mathrm{e}^{x^{\beta}} g_{\beta}(x)\right]^{\prime} } & =\mathrm{e}^{x^{\beta}}\left[g_{\beta}^{\prime}(x)+\beta x^{\beta-1} g_{\beta}(x)\right] \\
& =\mathrm{e}^{x^{\beta}}\left[q_{\beta}^{\prime}(x)+\beta x^{\beta-1} q_{\beta}(x)+1\right] .
\end{aligned}
$$

Therefore, if inequality (6) is valid, the function $\mathrm{e}^{x^{\beta}} g_{\beta}(x)$ is increasing. This implies Theorem 2 
Similar arguments lead to the following

Theorem 3. Define $f_{\theta}(x)$ as

$$
f_{\theta}(x)=\mathrm{e}^{x^{\theta}} \int_{0}^{x} \mathrm{e}^{-t^{\theta}} d t+r_{\theta}(x) \quad(\theta>0)
$$

where $\lim _{x \rightarrow 0} r_{\theta}(x)=0$ and

$$
r_{\theta}^{\prime}(x)-\theta x^{\theta-1} r_{\theta}(x)+1>0
$$

for $x>0$. Then $f_{\theta}(x)>0$. If $(8)$ is reversed, then $f_{\theta}(x)<0$.

\section{Particular inequalities}

3.1 Set $p_{\alpha}(x)=A x^{1-\alpha} \quad(A<0)$. Then

$$
\begin{aligned}
P_{\alpha}(x): & =p_{\alpha}^{\prime}(x)-\alpha x^{\alpha-1} p_{\alpha}(x)-1 \\
& =(1-\alpha) A x^{-\alpha}-(1+\alpha A) .
\end{aligned}
$$

For $\alpha>1$, if $A<-\frac{1}{\alpha}$, we get $P_{\alpha}(x)>0$, thus $h_{\alpha}(x)<0$. Hence, for $x>0$ and $\alpha>1$, the inequality

$$
\int_{x}^{\infty} \mathrm{e}^{-t^{\alpha}} d t<\frac{\mathrm{e}^{-x^{\alpha}}}{\alpha x^{\alpha-1}}
$$

holds.

For $0<\alpha<1$, if $A>-\frac{1}{\alpha}$, we have $P_{\alpha}(x)<0$ and $h_{\alpha}(x)>0$. Therefore, for $x>0$ and $0<\alpha<1$, inequality $(9)$ is reversed.

For $\alpha>1$, if $0>A>-\frac{1}{\alpha}$, when $x<\left(\frac{(1-\alpha) A}{1+\alpha A}\right)^{\frac{1}{\alpha}}$, we obtain

$$
\int_{x}^{\infty} \mathrm{e}^{-t^{\alpha}} d t<-A \frac{\mathrm{e}^{-x^{\alpha}}}{x^{\alpha-1}}
$$

when $x>\left(\frac{(1-\alpha) A}{1+\alpha A}\right)^{\frac{1}{\alpha}}$, inequality $(10)$ reverses.

For $0<\alpha<1$, if $A<-\frac{1}{\alpha}$, when $x<\left(\frac{(1-\alpha) A}{1+\alpha A}\right)^{\frac{1}{\alpha}}$, inequality (10) is reversed; when $x>\left(\frac{(1-\alpha) A}{1+\alpha A}\right)^{\frac{1}{\alpha}}$, inequality (10) holds.

3.2 Set $p_{\alpha}(x)=\frac{C}{(x+B)^{\alpha-1}}(B>0, C<0)$. Then

$$
\begin{aligned}
P_{\alpha}(x) & :=p_{\alpha}^{\prime}(x)-\alpha x^{\alpha-1} p_{\alpha}(x)-1 \\
& =\frac{(1-\alpha) C-\alpha C x^{\alpha-1}(x+B)-(x+B)^{\alpha}}{(x+B)^{\alpha}} .
\end{aligned}
$$


Let $\alpha=3$ and $B=1$. Then

$$
P_{3}(x)=\frac{-2 C-3 C x^{2}(x+1)-(x+1)^{3}}{(x+1)^{3}} .
$$

When $-\frac{1}{3}<C<0$, then $P_{\alpha}(x)<0$, thus $h_{\alpha}(x)>0$, that is

$$
\int_{x}^{\infty} \mathrm{e}^{-t^{3}} d t>\frac{\mathrm{e}^{-x^{3}}}{3(x+1)^{2}} \quad(x>0)
$$

Proposition 1. Denote $S_{n}(x)=\sum_{k=0}^{n} \frac{x^{k}}{k !}(x \in \mathbb{R})$, with $n>0$ odd. If $n \beta>1$, then for $x>0$ we have

$$
\frac{(n-1) !\left[\mathrm{e}^{x^{\beta}} S_{n-1}\left(-x^{\beta}\right)-1\right]}{\beta x^{n \beta-1}}<\int_{0}^{x} \mathrm{e}^{t^{\beta}} d t<\frac{n !\left[\mathrm{e}^{x^{\beta}} S_{n-1}\left(-x^{\beta}\right)-1\right]}{x^{n \beta-1}} .
$$

If $0<n \beta<1$, then inequality (12) is reversed.

Proof. Choose

$$
q_{\beta}(x)=\frac{E\left[\mathrm{e}^{-x^{\beta}}-S_{n-1}\left(-x^{\beta}\right)\right]}{x^{n \beta-1}}
$$

where $E>0$ is an undetermined constant. Direct computation produces

$$
q_{\beta}^{\prime}(x)=\frac{E\left\{\beta x^{\beta}\left[S_{n-2}\left(-x^{\beta}\right)-\mathrm{e}^{-x^{\beta}}\right]-(n \beta-1)\left[\mathrm{e}^{-x^{\beta}}-S_{n-1}\left(-x^{\beta}\right)\right]\right\}}{x^{n \beta}}
$$

and

$$
\begin{aligned}
Q_{\beta}(x) & :=q_{\beta}^{\prime}(x)+\beta x^{\beta-1} q_{\beta}(x)+1 \\
& =1+\frac{(-1)^{n} \beta}{(n-1) !} E-(n \beta-1) E\left(\frac{\mathrm{e}^{-x^{\beta}}-S_{n-1}\left(-x^{\beta}\right)}{x^{n \beta}}\right) .
\end{aligned}
$$

It is easy to prove that [6] the function $\frac{\mathrm{e}^{x}-S_{n}(x)}{x^{n+1}}$ is increasing for $x \in(0,+\infty)$ and

$$
\lim _{x \rightarrow 0} \frac{e^{x}-S_{n}(x)}{x^{n+1}}=\frac{1}{(n+1) !} .
$$

Therefore $Q_{\beta}(x)$ is monotone and

$$
\lim _{x \rightarrow 0} Q_{\beta}(x)=1+\frac{(-1)^{n}}{n !} E \quad \text { and } \quad \lim _{x \rightarrow+\infty} Q_{\beta}(x)=1+\frac{(-1)^{n} \beta}{(n-1) !} E .
$$

Now we have

$$
Q_{\beta}(x)\left\{\begin{array}{l}
>0 \text { if }\left\{\begin{array}{l}
n \beta>1 \text { and } E<\frac{(n-1) !}{\beta} \\
\text { or } \\
0<n \beta<1 \text { and } E<n !
\end{array}\right. \\
<0 \text { if }\left\{\begin{array}{l}
n \beta>1 \text { and } E>n ! \\
\text { or } \\
0<n \beta<1 \text { and } E>\frac{(n-1) !}{\beta} .
\end{array}\right.
\end{array}\right.
$$


Note that, if $n$ is an odd number, we have $\mathrm{e}^{x}-S_{n}(x) \geq 0 \quad(x \in \mathbb{R})$; if $n$ is an even number, this inequality holds for $x>0$ and reverses for $x \leq 0$ (this conclusion can be found in [1: p. 357] and [6]). Hence, for $n \beta>1$, we have

$$
\frac{(n-1) !\left[S_{n-1}\left(-x^{\beta}\right)-\mathrm{e}^{-x^{\beta}}\right]}{\beta x^{n \beta-1} \mathrm{e}^{-x^{\beta}}}<\int_{0}^{x} \mathrm{e}^{t^{\beta}} d t<\frac{n !\left[S_{n-1}\left(-x^{\beta}\right)-\mathrm{e}^{-x^{\beta}}\right]}{x^{n \beta-1} \mathrm{e}^{-x^{\beta}}}
$$

For $0<n \beta<1$, the reversed inequality of (13) holds. The proof of Proposition 1 is completed

\section{Proposition 2.}

(i) For $\theta>1$, we have

$$
\int_{0}^{x} \mathrm{e}^{-t^{\theta}} d t>\frac{1-\mathrm{e}^{-x^{\theta}}}{x^{\theta-1}} \quad(x>0)
$$

For $0<\theta<1$, inequality (14) reverses.

(ii) Let $n \geq 2$ be an integer. If $\frac{1}{n}<\theta<\frac{(n-1) !}{n !-1}$, the inequality

$$
\int_{0}^{x} \mathrm{e}^{-t^{\theta}} d t \geq \frac{(n-1) !}{\theta+(n-1) !(1-n \theta)} \cdot \frac{1-\mathrm{e}^{-x^{\theta}} S_{n-1}\left(x^{\theta}\right)}{x^{n \theta-1}} \quad(x>0)
$$

holds; if $0<n \theta<1$, the reversed inequality of (15) is sound.

Proof. Let $r_{\theta}(x)=D \frac{\mathrm{e}^{x^{\theta}}-1}{x^{\theta-1}}(x>0)$ and $D<0$ an undetermined constant. Then

$$
r_{\theta}^{\prime}(x)=D \frac{\left[\theta x^{\theta} \mathrm{e}^{x^{\theta}}+(\theta-1)\left(1-\mathrm{e}^{x^{\theta}}\right)\right]}{x^{\theta}}
$$

and

$$
\begin{aligned}
R_{\theta}(x): & =r_{\theta}^{\prime}(x)-\theta x^{\theta-1} r_{\theta}(x)+1 \\
& =1+\theta D+(\theta-1) D\left(\frac{1-\mathrm{e}^{x^{\theta}}}{x^{\theta}}\right) .
\end{aligned}
$$

When $\theta>1$, we get $R_{\theta}(x)>1+D$; when $0<\theta<1$, we have $R_{\theta}(x)<1+D$. Thus, inequality (14) is valid.

Let

$$
r_{\theta}(x)=F \frac{\mathrm{e}^{x^{\theta}}-S_{n-1}\left(x^{\theta}\right)}{x^{n \theta-1}} \quad(x>0 ; F<0, n \geq 2) .
$$

Straightforwardly calculating leads to

$$
r_{\theta}^{\prime}(x)=F \frac{\theta x^{\theta}\left[\mathrm{e}^{x^{\theta}}-S_{n-2}\left(x^{\theta}\right)\right]-(n \theta-1)\left[\mathrm{e}^{x^{\theta}}-S_{n-1}\left(x^{\theta}\right)\right]}{x^{\theta}}
$$


and

$$
\begin{aligned}
R_{\theta}(x):= & r_{\theta}^{\prime}(x)-\theta x^{\theta-1} r_{\theta}(x)+1 \\
& =1+\frac{\theta}{(n-1) !} F+(1-n \theta) F\left(\frac{\mathrm{e}^{x^{\theta}}-S_{n-1}\left(x^{\theta}\right)}{x^{n \theta}}\right) .
\end{aligned}
$$

When $n \theta-1>0$, we have

$$
R_{\theta}(x)>1+\frac{[\theta+(n-1) !(1-n \theta)]}{(n-1) !} F ;
$$

when $n \theta-1<0$, the above inequality is reversed. If

$$
n \theta-1>0, \quad \theta+(n-1) !(1-n \theta)>0, \quad F>\frac{(n-1) !}{(n-1) !(n \theta-1)-\theta},
$$

we obtain $R_{\theta}(x)>0$. From this, it is deduced that inequality (15) holds. The proof of Proposition 2 is completed

Remark. The results in this paper generalize, extend or refine those in $[2,7]$. However, the results in this paper and those in $[5,9]$ are not included each other.

Acknowledgements. The authors are indebted to the referees for their helpful and valuable suggestions and corrections.

\section{References}

[1] Ji-Chang Kuang: Applied Inequalities (in Chinese), 2nd edition. Changsha (China): Hunan Education Press 1993.

[2] Laforgia, A. and S. Sismondi: Monotonicity results and inequalities for the gamma and error functions. J. Comp. Appl. Math. 23 (1988), 25 - 33.

[3] Mitrinović, D. S.: Analytic Inequalities. New York et al.: Springer-Verlag 1970.

[4] Feng Qi: Generalized weighted mean values with two parameters. Proc. Roy. Soc. London (Ser. A) 454 (1998), 2723 - 2732.

[5] Feng Qi: Monotonicity results and inequalities for the gamma and incomplete gamma functions. Math. Ineq. Appl. (2000) (to appear).

[6] Feng Qi: A method of constructing inequalities about $\mathrm{e}^{x}$. Univ. Beograd. Publ. Elektrotehn. Fak. Ser. Mat. 8 (1997), $16-23$.

[7] Feng Qi, Li-Hong Cui and Sen-Lin Xu: Some inequalities constructed by Tchebycheff's integral inequality. Math. Ineq. Appl. 1999 (to appear).

[8] Feng Qi and Bai-Ni Guo: Inequalities for generalized weighted mean values of convex function. Archiv Math. (to appear).

[9] Feng Qi and Sen-Lin Guo: Inequalities for the incomplete gamma and related functions. Math. Ineq. Appl. 2 (1999), $47-53$.

[10] Feng Qi and Shi-Qin Zhang: Note on monotonicity of generalized weighted mean values. Proc. Roy. Soc. London (Ser. A) (1999) (to appear). 